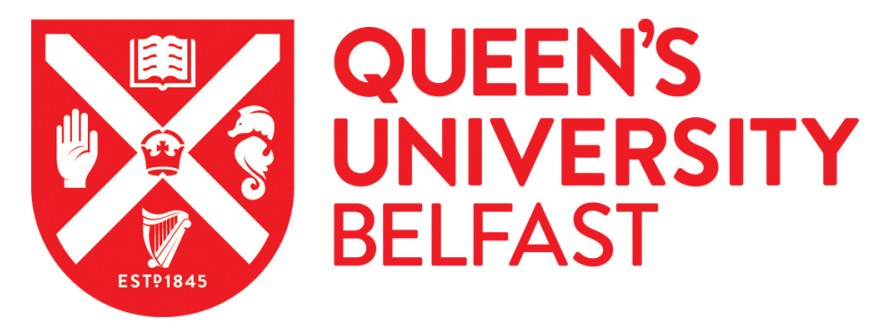

\title{
Inter-Laboratory Evaluation of a Next-Generation Sequencing Panel for Acute Myeloid Leukemia
}

Haslam, K., Catherwood, M. A., Dobbin, E., Sproul, A., Langabeer, S. E., \& Mills, K. I. (2016). Inter-Laboratory Evaluation of a Next-Generation Sequencing Panel for Acute Myeloid Leukemia. Molecular Diagnosis \& Therapy, 20(5), 457-461. https://doi.org/10.1007/s40291-016-0222-3

Published in:

Molecular Diagnosis \& Therapy

Document Version:

Peer reviewed version

Queen's University Belfast - Research Portal:

Link to publication record in Queen's University Belfast Research Portal

Publisher rights

(c) 2016 Springer

The final publication is available at Springer via http://dx.doi.org/10.1007/s40291-016-0222-3

\section{General rights}

Copyright for the publications made accessible via the Queen's University Belfast Research Portal is retained by the author(s) and / or other copyright owners and it is a condition of accessing these publications that users recognise and abide by the legal requirements associated with these rights.

Take down policy

The Research Portal is Queen's institutional repository that provides access to Queen's research output. Every effort has been made to ensure that content in the Research Portal does not infringe any person's rights, or applicable UK laws. If you discover content in the Research Portal that you believe breaches copyright or violates any law, please contact openaccess@qub.ac.uk. 


\section{Inter-laboratory evaluation of a next-generation sequencing panel for acute myeloid leukemia}

Karl Haslam ${ }^{1}$, Mark A. Catherwood ${ }^{2}$, Edwina Dobbin ${ }^{3}$, Anne Sproul ${ }^{3}$, Stephen E. Langabeer ${ }^{1}$, Ken I. Mills ${ }^{2}$

${ }^{1}$ Cancer Molecular Diagnostics, St. James’s Hospital, Dublin, Ireland

${ }^{2}$ Centre for Cancer Research \& Cell Biology, Queens University, Belfast, United Kingdom

${ }^{3}$ Department of Haematology, Western General Hospital, Edinburgh, United Kingdom

Key words: acute myeloid leukemia, next generation sequencing, inter-laboratory evaluation

Word count: 1560

\section{Corresponding author:}

Karl Haslam

Cancer Molecular Diagnostics

Central Pathology Laboratory

St. James’s Hospital

Dublin 8

Ireland

Tel: +35314103567

Fax: +35314103513_E-mail: khaslam@stjames.ie 


\section{Abstract}

Introduction Acute myeloid leukemia (AML) is a heterogeneous clonal disorder often associated with dismal overall survival. The clinical diversity of AML is reflected in the range of recurrent somatic mutations in several genes, many of which have a prognostic and therapeutic value. Targeted next-generation sequencing of these genes has potential for translation into clinical practice. In order to assess this potential, an inter-laboratory evaluation of a commercially available AML gene panel across three diagnostic centres in the UK and Ireland was performed.

Methods DNA from six AML patient samples was distributed to each centre and processed using a standardised workflow, including a common sequencing platform, sequencing chips and bioinformatics pipeline. A duplicate sample in each centre was run to assess intralaboratory performance.

Results An average sample read depth of 2725X (range 629-5600) was achieved using six samples per chip, with some variability observed in the depth of coverage generated for individual samples and between centres. A total of 16 somatic mutations were detected in the six AML samples with a mean of 2.7 mutations per sample (range 1-4) representing nine genes on the panel. Allelic frequencies of the mutations ranged from 5.6\% - 53.3\% (median 44.4\%) with a high level of concordance of these frequencies between centres for mutations detected.

Conclusion In this inter-laboratory comparison, a high concordance, reproducibility and robustness was demonstrated using a commercially available next-generation sequencing AML gene panel and platform. 


\section{Introduction}

Acute myeloid leukemia (AML) is a heterogeneous clonal disorder characterised by infiltration of the bone marrow, blood and other tissues by abnormal or poorly differentiated myeloid cells of the hematopoietic system. Diagnosis is based on bone marrow and peripheral blood morphology, immunophenotype and the presence of typical chromosomal alterations or recurrent mutations. Treatment for AML patients is primarily based on delivery of an intensive course of induction chemotherapy such as cytarabine plus anthracycline followed by post remission consolidation chemotherapy. Up to $80 \%$ of patients achieve complete remission however a significant proportion will eventually relapse [1]. Allogeneic transplantation offers the potential of a curative therapy in eligible patients after careful assessment of co-existing morbidities based on donor availability and genetic profile. Recurrent genetic lesions such as Fms-related tyrosine kinase 3 (FLT3), CCAAT/enhancer binding protein- $\alpha(C E B P A)$ and nucleophosmin (NPM1) are commonly used as prognostic markers in clinical practice, with markers associated with poor outcome such as tumour protein 53 (TP53), additional sex combs like transcriptional regulator (ASXL1) and runtrelated transcription factor 1 (RUNX1) likely to be recommended for stratification in forthcoming guidelines [2].

Next generation sequencing (NGS) technologies allow massive parallel sequencing of multiple DNA regions of interest including large or GC rich genes and offer the potential for increased throughput, sensitivity and quantification of variant allelic frequencies relative to standard Sanger sequencing. Recent application of genomic techniques such as whole genome sequencing have yielded a broad view of the mutational landscape of AML and highlighted 25-30 recurrent somatic mutations that can be organised into functional categories. These genes encode for transcription factors and tumor suppressors, genes related to DNA methylation, hematopoietic cytokine signalling, chromatin modification, the cohesin 
complex, and the spliceosome complex [3]. Further studies have described patterns of clonal and subclonal evolution, mutation clearance rates post induction therapy and the monitoring of minimal residual disease [4-7]. Together these advances are helping unravel the potential for new personalised therapies and targeted treatments with specific agents used in combinations with standard chemotherapy to maintain remission or bridge to transplantation [2,8]. The adoption of targeted gene panels comprising a refined set of disease relevant genes has shown promise for translation of NGS into clinical practice [9-11]. Here we present an inter-laboratory evaluation of a commercially available AML gene panel across three clinical diagnostic centres in the UK and Ireland.

\section{Material and Methods}

To evaluate the AML NGS panel, DNA from six pre-characterised AML patients (NPM1 and FLT3-ITD mutation status assessed by polymerase chain reaction [PCR] and capillary electrophoresis using alternative laboratory methods) was used for quality control (QC\#1-6), distributed to each centre and processed using standardised workflows for the Ion AmpliseqTM AML Panel (Life Technologies/Thermo Fisher Scientific, Paisley, UK) before sequencing on an Ion Torrent Personalized Genome Machine (PGMTM, Ion TorrentTM, Life Technologies/Thermo Fisher Scientific, Paisley, UK). A designated duplicate sample (QC\#3, QC\#5, and QC\#6) was repeated on separate runs in centres 1, 3 and 2 respectively to assess intra-laboratory reproducibility. Amplicon libraries covering 19 commonly mutated genes mplicated in AML and covering the entire coding region of DNA (cytosine-5-)methyltransferase 3-a (DNMT3A), CEBPA, GATA binding protein 2 (GATA2), tet methylcytosine dioxygenase 2 (TET2), TP53 and mutational hot-spot regions of ASXL1, Braf proto-oncogene, serine/threonine kinase (BRAF), Cbl proto-oncogene, E3 ubiquitin protein ligase (CBL), FLT3 (tyrosine kinase domain mutations), isocitrate dehydrogenase 1 (IDH1), isocitrate dehydrogenase 2 (IDH2), Janus kinase 2 (JAK2), v-kit Hardy-Zuckerman 4 
feline sarcoma viral oncogene homolog (KIT), Kirsten rat sarcoma viral oncogene homolog (KRAS), NPM1, neuroblastoma RAS viral (v-ras) oncogene homolog (NRAS), protein tyrosine phosphatase, non-receptor type 11 (PTPN11), RUNX1 and Wilms tumour 1 (WT1), were generated using $10 \mathrm{ng}$ genomic DNA in each of four primer-pooled reactions generating 264 amplicons.

Amplified targets were combined from four pools to two, primer sequences were partly digested and barcoded adapters were ligated for sample identification. The resulting product was purified using Agencourt ${ }^{\circledR}$ AMPure ${ }^{\circledR}$ XP beads as per manufacturer's instruction (Beckman Coulter, Brea, CA, USA). The unamplified libraries were quantified by qPCR with the Ion Library Quantification Kit (Life Technologies/Thermo Fisher Scientific, Paisley, UK) and equalised to 100pM prior to combination. Emulsion PCR, recovery and enrichment of templated Ion Sphere ${ }^{\mathrm{TM}}$ particles (ISP) was performed using the Ion OneTouch $^{\mathrm{TM}} 2$ System or Ion Chef ${ }^{\mathrm{TM}}$ System (automated), before loading onto Ion $318^{\mathrm{TM}} \mathrm{v} 2$ chips and sequencing on the Ion Torrent $\mathrm{PGM}^{\mathrm{TM}}$ platform with the Ion $\mathrm{PGM}^{\mathrm{TM}}$ Sequencing 200 kit v2. Data was reviewed using Torrent Suite ${ }^{\mathrm{TM}}$ software v4.2.1.0 (Thermo Fisher Scientific, Life Technologies, Paisley, UK) using a low stringency parameter configuration. Samples were uploaded to Ion Reporter ${ }^{\mathrm{TM}}$ v4.2 cloud based software (Thermo Fisher Scientific, Life Technologies, Paisley, UK) as BAM or VCF files for subsequent variant annotation.

Coverage in highly sampled locations was capped at 2000X to reduce computational time. Confident calling of somatic mutations was achieved using an algorithm that excluded homozygous or synonymous mutations, variants located within intronic or un-translated regions and variants present at a frequency of $<5 \%$. Insertions/deletions called using Ion Reporter $^{\mathrm{TM}}$ were confirmed by reviewing data with the Broad Institute's Integrative Genomics Viewer (IGV) [12]. In addition, AcroMetrix controls (Life Technologies/Thermo 
Fisher Scientific), a highly multiplexed QC used to assess the performance of NGS assays, was employed in one centre to determine variant detection at low variant allele frequencies (VAFs). To maximise confidence in variant calling, a minimum target depth of coverage for variant calls was set at 500X based on these data, with potential somatic variants reviewed and screened using relevant public databases, such as dbSNP, ClinVar, COSMIC, and PubMed. No homozygous mutations of somatic relevance were detected using the aforementioned public databases.

\section{Results}

\subsection{Platform performance}

Performance of the Ion Torrent platform and workflow at each centre was assessed using the quality control data generated for each sample (Supplementary Table 1). Variables assessed were the number of reads generated, the number and AQ20 (1\% error rate) quality score of bases, the average depth of coverage across the bases and the average read length generated. A mean read count of 602,354 reads per sample (range 150,312-1,212,941) was generated. Mean read length at AQ20 was consistent at 123 bp (CV 3.3\%) for all samples analysed. An average sample read depth of 2725X (range 629-5600) was achieved using 6 samples on a $318^{\mathrm{TM}} \mathrm{v} 2$ chip, with significant variability observed in the depth of coverage generated for

individual QC samples and between centres (electronic supplementary Table 1). AcroMetrix controls showed detection of applicable variants present in IDH1/2, KIT, NPM1, PTPN11, FLT3, TP53, KRAS and NRAS at VAFs of between 5 and $10 \%$ (25 \% for NPM1) at 500X as expected,

\subsection{Somatic mutation detection}

Unfiltered data files from each centre were downloaded from Ion Reporter ${ }^{\mathrm{TM}}$ and analysed using the filtering algorithm outlined above. A total of 16 somatic mutations were identified in the six AML patient samples tested. Centres 1 and 3 identified all 16 mutations, whereas 
Centre 2 identified 15/16 producing analytical specificity for the gene panel of 97.9\% (95\% CI 89-99\%)(electronic supplementary Table 2). A mean of 2.7 somatic mutations were detected in each AML patient sample (range 2-6) representing 10 genes on the panel (DNMT3A, TET2, NPM1, NRAS, RUNX1, KIT, PTPN11, ASXL1, IDH1, IDH2) with TET2 and DNMT3A being the most frequent. NPM1 mutations were identified in one of the siz QC samples confirming results obtained at diagnosis by standard methodology at the referring centre. In addition two frameshift deletions in ASXL1 not currently represented in COSMIC were identified in all centres and confirmed using IGV.

Allelic frequencies of the 16 mutations detected ranged from 5.6 - 53.3\% (median 44.4\%) with a high concordance between centres for mutations detected (Figure 1). Of note, Centre 2 did not report detection of the PTPN11 c.215C>A p.Ala72Asp mutation in sample three. Reanalysis of the data confirmed the presence of the missed mutation at a read depth of 302X, initially omitted as the read depth of 500X (in italics in electronic supplementary Table 2 and Fig 1, mutation number 7.

.In addition one mutation, a $R U N X 1$ c.167T $>C$ p.Leu56Ser missense mutation detected in QC\#4 showed a difference in allelic frequency (Figure 2 mutation 17), with Centre 1 and 3 detecting the mutation at approximately 50\% compared to 24\% for Centre 2 despite deep coverage ( $>1000$ fold) of the sample at this site by all centres. Assessment of intra-laboratory reproducibility using duplicate samples showed concordance with results generated at all centres (Supplementary Table 2).

\subsection{Reproducibility}

Analysis of duplicate sample data showed both within and between run concordances, with the same number of variants detected at similar frequencies by all centres (electronic supplementary Table 2). 


\section{Discussion}

There is considerable interest in translating targeted NGS of AML into clinical laboratories for routine detection of therapeutically actionable disease mutations $[10,11,13,14]$. In this report, we have evaluated the inter- and intra-laboratory reproducibility and robustness of a commercial 19-gene AML panel in three separate clinical centres in the UKand Ireland. Performance of the panel was assessed using six AML patient samples run on the Ion PGMTM platform across all centres.Ahigh level of concordance was observed, with 15/16 mutations detected. Centre 2 did not report a PTPN11 c.215C[A p.Ala72Asp mutation that was excluded as the number of reads generated for the sample was low $(150,312)$ and the subsequent read depth at the target site did not achieve 500X. As a depth of coverage of 500X is necessary for confidence in calling low-level mutations (25 mutated reads are required for a $5 \%$ burden), it is therefore essential to ensure appropriate coverage of all relevant amplicons to enable appropriate interrogation and interpretation of data produced. Improving efficiencies during the preanalytic stages could help achieve this and would reduce the variability in reads generated between samples and between centres. Optimisation of library equalisation or implementation of automated template preparation and chip loading (Ion ChefTM) could increase reads generated and improve preanalytical consistency. Reduction of sample number per chip or repeat testing and combination of results for samples that prove difficult to sequence would help deliver sufficient read depth for further analysis.

Nevertheless, given the variation in reads generated per sample by each centre, the high concordance in the identification and coverage depth of somatic mutations in the samples tested proves the capacity of the Ion AmpliseqTM AML Research Panel and Ion Torrent as a robust platform for generating reproducible results in a clinical diagnostic setting.

Incorporation of this gene panel would complement and enhance the armoury of tools available for identification of relevant diagnostic and prognostic somatic mutations in AML 
patients. A limitation of this study was the limited number of genes available for analysis. Expansion of the panel to incorporate a greater number of relevant genes mutated in AML (e.g. splicing factors) would further improve its applicability.

\section{Conclusion}

The use of targeted panels in AML has the potential to overcome the limitations of standard Sanger sequencing in terms of time, the potential to sequence multiple gene targets at once and the ability to quantify variant allelic frequencies. In a clinical diagnostic setting these benefits translate into additional clinically relevant information and justify the application of limited resources to generate relevant information for clinical decision making. The implementation of common platforms and workflows to interrogate the mutational profile of AML patients will enable standardisation of results and further collaborative efforts between centres.

\section{Compliance with Ethical Standards}

Conflict of interest Karl Haslam, Mark A. Catherwood, Edwina Dobbin, Anne Sproul, Stephen E. Langabeer and Ken I. Mills declare that they have no competing interests. Funding Ken I. Mills was employed by Queen's University Belfast, and part of this work was supported from the development Grant (R2536CNR) awarded from Leukaemia and Lymphoma Northern Ireland.

Ethical Approval and Informed Consent All procedures performed involving human participants were in accordance with the ethical standards of the institution and the 1964 Helsinki declaration or comparable ethical standards. 


\section{References}

[1] Buccisano F, Maurillo L, Del Principe MI, Del Poeta G, Sconocchia G, Lo-Coco F, et al. Prognostic and therapeutic implications of minimal residual disease detection in acute myeloid leukemia. Blood. 2012;119(2):332-41.

[2] Dohner H, Weisdorf DJ, Bloomfield CD. Acute Myeloid Leukemia. N Engl J Med. 2015;373(12):1136-52.

[3] The Cancer Genome Atlas Network. Genomic and epigenomic landscapes of adult de novo acute myeloid leukemia. N Engl J Med. 2013;368(22):2059-74.

[4] Welch JS, Ley TJ, Link DC, Miller CA, Larson DE, Koboldt DC, et al. The origin and evolution of mutations in acute myeloid leukemia. Cell. 2012;150(2):264-78.

[5] Klco JM, Miller CA, Griffith M, Petti A, Spencer DH, Ketkar-Kulkarni S, et al. Association between mutation clearance after induction therapy and outcomes in acute myeloid leukemia. JAMA. 2015;314(8):811-22.

[6] Debarri H, Lebon D, Roumier C, Cheok M, Marceau-Renaut A, Nibourel O, et al. IDH1/2 but not DNMT3A mutations are suitable targets for minimal residual disease monitoring in acute myeloid leukemia patients: a study by the Acute Leukemia French Association. Oncotarget. 2015;6(39):42345-53.

[7] Ivey A, Hills RK, Simpson MA, Jovanovic JV, Gilkes A, Grech A, et al. Assessment of minimal residual disease in standard-risk AML. N Engl J Med. 2016;374(5):42233.

[8] Falini B, Sportoletti P, Brunetti L, Martelli MP. Perspectives for therapeutic targeting of gene mutations in acute myeloid leukaemia with normal cytogenetics. $\mathrm{Br} \mathrm{J}$ Haematol. 2015;170(3):305-22.

[9] Kohlmann, A, Grossmann V, Nadarajah N, Haferlach T. Next-generation sequencing - feasibility and practicality in haematology. Br J Haematol. 2014;160(6):736-53. 
[10] McCourt CM, McArt DG, Mills K, Catherwood MA, Maxwell P, Waugh DJ. et al. Validation of next generation sequencing technologies in comparison to current diagnostic gold standards for BRAF, EGFR, KRAS mutational analysis. PLoS One. 2013;8(7):e69604.

[11] Ivanova M, Shivarov V, Pavlov I, Lilakos K, Naumova E. Clinical evaluation of a novel nine-gene panel for Ion Torrent PGM sequencing of myeloid malignancies. Mol Diagn Ther. 2016;20(1):27-32.

[12] Robinson JT, Thorvaldsdóttir H, Winckler W, Guttman M, Lander ES, Getz G, Mesirov JP. Integrative Genomics Viewer. Nat Biotechnol. 2011;29(1):24-6.

[13] Kohlmann A, Klein HU, Weissmann S, Bresolin S, Chaplin T, Cuppens H, et al. The Interlaboratory Robustness of Next-generation sequencing (IRON) study: a deep sequencing investigation of TET2, CBL, and KRAS mutations by an international consortium involving 10 laboratories. Leukemia 2011;25(12):1840-8.

[14] Grossmann V, Roller A, Klein HU, Weissmann S, Kern W, Haferlach C, et al. Robustness of amplicon deep sequencing underlines its utility in clinical applications. J Mol Diagn. 2014;15(4):473-84. 


\section{Figure legends:}

Figure 1: Allelic frequency of mutations detected in quality-control samples. Mutation 1: DNMT3A p.Leu344Gln; Mutation 2: NPM1 p.Trp288fs; Mutation 3: NRAS p.Gly12Asp; Mutation 4: NRAS p.Gly12Ser; Mutation 5: TET2 pArg1216Ter; Mutation 6: RUNX1 p.Arg204Gln; Mutation 7: PTPN11 p.Ala72Asp; Mutation 8: ASXL1 p.Leu890fs; Mutation

9: DNMT3A p.Arg882His; Mutation 10: IDH1 p.Arg132Cys; Mutation 11: ASXL1 p.Arg693Ter; Mutation 12: ASXL1 p.Gly966del; Mutation 13: DNMT3A p.Arg882Cys; Mutation 14: DNMT3A p.Arg792His; Mutation 15: IDH2, p.Arg172Lys; Mutation 16: ASXL1 p.Ser798fs

\section{Supplementary table legends:}

Supplementary Table 1: Performance variables for Ion Torrent Ampliseq AML Panel across six QC samples.

Supplementary Table 2: Somatic mutations identified in six QC samples. 


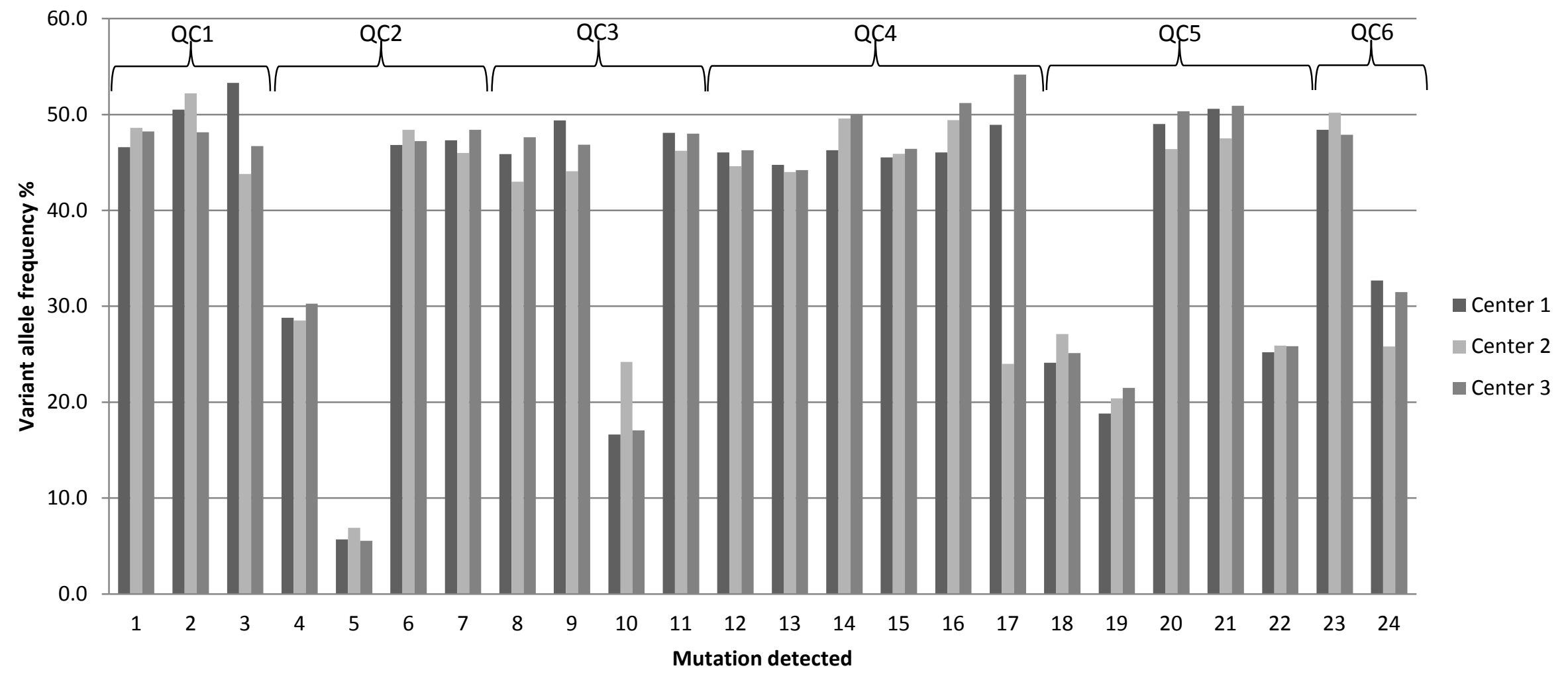

